\title{
Global strings in five-dimensional supergravity
}

\author{
Miho Marui and Kiyoshi Shiraishi \\ Department of Physics, Faculty of Science, Ochanomizu University, \\ 1-1 Otsuka 2, Bunkyou-ku, Tokyo 112, Japan \\ Physcs Letters B259 (1991) 58-62
}

\begin{abstract}
We show the existence of solitonic solutions of five-dimensional supergravity, which can be interpreted as global cosmic strings in our universe. They possess the same mathematical structure as the stringy cosmic strings studied by Greene, Shapere, Vafa and Yau, while the size of the extra space and the value of the extra-space component of the gauge field vary from place to place around the string in our model. We also show that supersymmetry is partially broken in the presence of the global strings.
\end{abstract}

Classical static solutions of higher-dimensional theories including gravity have been studied extensively by many authors during the past decade (see ref. [1] on higher-dimensional "black holes", ref. [2] on monopoles, and ref. [3] on cosmic strings). In the present paper, we examine some classical solutions of the $\mathcal{N}=2, D=5$ supergravity theory $[4,5]$, which can be derived from $\mathcal{N}=1$, $D=11$ supergravity [6], by dimensional reduction.

Recently, Greene, Shapere, Vafa and Yau studied [7] vortex-like solutions in the system of multi-dimensional gravity plus complex scalar fields, whose kinetic terms are non-canonical. We can identify the scalar fields with the moduli of an extra torus space. The scalar fields can also be interpreted as combinations of dilaton and antisymmetric fields which are naturally introduced when we consider string theories. They also discussed the global structure of space in which propagation of strings is admitted. Dabholkar et al. [8] and Strominger [9] studied another class of solitonic solutions in string theory and discussed supersymmetry in the background of the topological object.

"Strings" in our five-dimensional model belong to the same type as studied by Greene et al. [7]. It is outstanding that the solution of moduli is given by an arbitrary holomorphic function. A simple model that they offered in their paper is a six-dimensional model. We consider five-dimensional supergravity in the present paper. One of the aims of this paper is to provide the simplest, pedagogical model which realizes similar solutions. Another aim is to discuss supersymmetry in the presence of the string in the specified model. While the analysis is very similar to refs. [8, 9], only holomorphicity of "moduli" is needed in the present analysis. 
We begin with the five-dimensional $\mathcal{N}=2$ supergravity theory. The supermultiplet consists of the fünfbein $e_{M}^{A}\left(x^{N}\right)$, the gravitino $\psi_{M}^{a}\left(x^{N}\right)$ (where $a=1,2$ ), and the gauge field $A_{M}\left(x^{N}\right)$. Our notation is almost the same as that of ref. [5]. To make the present paper self-contained, however, we shall exhibit the notational convention.

One extra dimension is compactified on $S^{1}$, i.e., five-dimensional coordinates are separated as $x^{M}=\left(x^{\mu}, x^{5}\right)=\left(x^{\mu}, \theta\right)$ with $0 \leq \theta<2 \pi$. We use $M, N, \ldots(=\dot{1}, \ldots, \dot{5}), \mu, \nu, \ldots(=\dot{1}, \ldots, \dot{4})$, for world indices and $A, B, \ldots(=$ $1, \ldots, 5), \alpha, \beta, \ldots(=1, \ldots, 4)$ for Lorentz indices.

The supersymmetric action is

$$
\begin{aligned}
S^{(5)}= & \int d^{5} x\left(-\frac{1}{4} e e_{A}^{M} e_{B}^{N} R^{A B}{ }_{M N}-\frac{1}{4} e F_{M N} F^{M N}\right. \\
& -\frac{1}{6 \sqrt{3}} \epsilon^{M N P S L} F_{M N} F_{P S} A_{L}-\frac{1}{2} i e \bar{\psi}_{M}^{a} \Gamma^{M N P} D_{N}(\omega+\hat{\omega}) \psi_{P}^{a} \\
& \left.-\frac{1}{32} i \sqrt{3} e\left(F_{M N}+\hat{F}_{M N}\right) \bar{\psi}^{P a}\left(\gamma_{p} \Gamma^{M N} \gamma_{S}-\gamma_{S} \Gamma^{M N} \gamma_{P}\right) \psi_{a}^{S}\right) .
\end{aligned}
$$

The "generalized Majorana spinor" $\psi_{M}^{a}$, the covariant derivative $D_{N}$, the totally antisymmetrized $\gamma$-matrices $\Gamma^{A B \cdots}, \hat{F}_{M N}$, and $\hat{\omega}_{M A B}$ are defined as follows:

$$
\bar{\psi}_{M}^{a}=\psi_{M}^{a} C, \quad \bar{\psi}_{M}^{1}=\psi_{M}^{2}{ }^{*} \gamma^{0}, \quad \bar{\psi}_{M}^{2}=-\psi_{M}^{1}{ }^{*} \gamma^{0},
$$

where $C=i \gamma_{1} \gamma^{5}$ is the charge-conjugation matrix,

$$
\begin{aligned}
& D_{N}[\omega] \psi_{P a}=\partial_{N} \psi_{P a}+\frac{1}{4} \omega_{N P S} \psi_{a}^{S}, \\
& \Gamma^{A_{1} \cdots A_{N}}=\frac{1}{N !} \sum_{\text {perm. }\left(A_{1} \cdots A_{N}\right)} \operatorname{sgn}\left(A_{1} \cdots A_{N}\right) \gamma^{A_{1}} \cdots \gamma^{A_{N}}, \\
& \hat{F}_{M N}=F_{M N}+\frac{1}{2} i \sqrt{3} \bar{\psi}_{M}^{a} \psi_{N a}, \\
& \hat{\omega}_{M A B}=\omega_{M A B}+\frac{1}{4} i \bar{\psi}^{P a} \Gamma_{M A B P S} \psi_{a}^{S} .
\end{aligned}
$$

To construct a string-like classical solution, we give the following vacuum configurations of fields:

$$
\begin{aligned}
& \left\langle e_{\mu}^{\alpha}\right\rangle=\frac{1}{\sqrt{b}} \bar{e}_{\mu}^{\alpha}\left(x^{\mu}\right), \quad\left\langle e_{\dot{5}}^{5}\right\rangle=b\left(x^{\mu}\right), \\
& \left\langle A_{\dot{5}}\right\rangle=A_{\dot{5}}\left(x^{\mu}\right), \\
& \langle\text { all other fields }\rangle=0,
\end{aligned}
$$

where $b$ means the radius of $S^{1}$, the extra space. These vacuum configurations are chosen to be consistent with the equations of motion derived from the action (1) in the tree approximation. The configuration involving non-trivial dependence on the spatial coordinates is very interesting to us. To analyze 
such configurations, we look into the relevant part of the four-dimensional action originating from the Einstein-Maxwell system in five dimensions through dimensional reduction:

$$
S_{e f f}^{(4)}=2 \pi \int d^{4} x \frac{-\bar{e}}{4}\left(\bar{R}^{(4)}-\frac{3}{2} \frac{\bar{\nabla}_{\mu} b \bar{\nabla}^{\mu} b}{b^{2}}-2 \frac{\bar{\nabla}_{\mu} A_{\dot{5}} \bar{\nabla}^{\mu} A_{\dot{5}}}{b^{2}}\right),
$$

where letters with overbar indicate that those are defined in terms of $\bar{e}_{\mu}^{\alpha}$ in eq. (7).

We define a complex scalar field ("moduli") $\tau$, that is,

$$
\tau=\tau_{1}+i \tau_{2}, \text { where } \tau_{1}=A_{\dot{5}} \text { and } \tau_{2}=\frac{1}{2} \sqrt{3} b
$$

By the use of (11), the effective action is written as

$$
S_{e f f}^{(4)}=2 \pi \int d^{4} x \frac{-\bar{e}}{4}\left(\bar{R}^{(4)}+\frac{3}{8} \frac{\bar{\nabla}_{\mu} \tau \bar{\nabla}^{\mu} \tau}{(\tau-\bar{\tau})^{2}}\right) .
$$

This action resembles one discussed by Greene et al. [7], up to a coefficient in the kinetic term of $\tau$. They considered torus compactification in six-dimensional theory as a simple example. They took an ansatz for the complex scalar field $\tau$ (moduli of the two-torus)

$$
\tau=\tau\left(x^{2}, x^{3}\right),
$$

and that for the metric of the four-dimensional theory

$$
\bar{d} s^{2}=\bar{g}_{\mu \nu} d x^{\mu} d x^{\nu}=\left(d x^{1}\right)^{2}-e^{\phi}\left[\left(d x^{2}\right)^{2}+\left(d x^{3}\right)^{2}\right]-\left(d x^{4}\right)^{2},
$$

where $\phi=\phi\left(x^{2}, x^{3}\right)$. The metric of this form indicates that the configuration is homogeneous along with the $x^{4}$-direction. Greene et al. solved the coupled Einstein equations in terms of the above variables and then found a cosmic string solution dubbed as a "stringy cosmic string" [7].

Now we adopt their assumptions (13) and (14) in our model. The equation of motion for $\tau$ takes the same form as theirs, i.e.,

$$
\partial \bar{\partial} \tau+\frac{2 \partial \tau \bar{\partial} \bar{\tau}}{\tau-\bar{\tau}}=0
$$

while the non-trivial Einstein equation turns out to be

$$
\partial \bar{\partial} \phi=\frac{3}{(\tau-\bar{\tau})^{2}}(\partial \tau \bar{\partial} \bar{\tau}+\bar{\partial} \tau \partial \bar{\tau}),
$$

where $\partial \equiv \partial / \partial z$ and $\bar{\partial} \equiv \partial / \partial \bar{z}$, for $z=x^{2}+i x^{3}$ and $\bar{z}=x^{2}-i x^{3}$.

Any holomorphic (or anti-holomorphic) function $\tau$ is a solution to (15), that is,

$$
\bar{\partial} \tau=0 \quad(\text { or } \partial \tau=0)
$$


We concentrate on the holomorphic solution of this type in the present paper. Substituting (17) into (16) then gives

$$
\partial \bar{\partial} \phi=3 \partial \bar{\partial} \ln \tau_{2} .
$$

The solution to eq. (18) is therefore obtained as

$$
\phi(z, \bar{z})=\ln \tau_{2}^{3}(z, \bar{z})+\ln f(z)+\ln f(\bar{z}),
$$

where $f(z)$ is some regular function.

From the observations so far, we can conclude that the string-like solution, which is very similar to the "stringy string" obtained in ref. [7], can be constructed in terms of $\tau$ and $\phi$ in five-dimensional supergravity. The only (mathematical) difference is the powers of $\tau$ in eq. (19), which comes from the coefficient 3 in eqs. (16) and (18).

We should note that $\tau+1$ is equivalent to $\tau$ because the only local gauge equivalence permitted in the fifth dimension is the identification $A_{\dot{5}} \sim A_{\dot{5}}+1$ in the periodic dimension [10].

As in ref. [7], we can choose the Jacobi function for $\tau$ in order to obtain a string solution with finite energy density per unit length. In our model, we find a discontinuity in $A_{5}$ at the boundary of the fundamental region, $|\tau|=1$. The appearance of singularities in the values of the fields is a generic feature of vortices in non-linear sigma models [11]. Moreover the discontinuity in our model can be avoided if we consider $S^{1} / Z_{2}$ as extra space, where the gauge fields of opposite signs are identified with each other. We leave the discussion on the property of an explicit solution written in terms of elliptic functions for other occasions. In the rest of this paper, we discuss a property of the string background without an explicit functional form of the solution.

Let us discuss the supersymmetric structure of the string-like configuration in five-dimensional supergravity. Infinitesimal transformations of the supersymmetry on five-dimensional fields are as follows [5]:

$$
\begin{aligned}
\Delta e_{M}^{A} & =-i \bar{\epsilon} \gamma^{A} \psi_{M}, \\
\Delta \psi_{M} & =\partial_{M} \epsilon+\frac{1}{4} \hat{\omega}_{M A C} \Gamma^{A C} \epsilon+\frac{1}{4 \sqrt{3}}\left(\Gamma_{M}^{P Q}+4 \gamma^{P} \delta_{M}^{Q}\right) \hat{F}_{P Q} \epsilon \\
\Delta A_{M} & =-\frac{1}{2} i \sqrt{3} \bar{\epsilon} \psi_{M},
\end{aligned}
$$

where indices $a(=1,2)$ of $\epsilon$ and $\psi$ are implicit.

In the dimensionally reduced theory, we wish to concentrate our attention on zero modes, because a matter of interest to us is the vacuum configuration and the zero modes corresponding to unbroken symmetries. It is easy to see that the transformation of bosonic fields in the string background is zero, for the fermionic vacuum configuration is absent. On the other hand, the transformation of does not vanish in general; thus we must investigate what form of satisfies the equation $\left\langle\Delta \psi_{M}\right\rangle=0$, as in refs. $[8,9]$.

Note that we can treat $\epsilon$ as a complex spinor instead of two "generalized Majorana" spinors. Hereafter we forget the label on $\epsilon$. 
By substituting eqs. (7), (8), (9), (13) and (14) into eq. (21) and using holomorphicity of $\tau$, i.e., the Cauchy-Riemann equation on $\tau$ (eq. (17)), we obtain

$$
\begin{aligned}
\left\langle\Delta \psi_{i}\right\rangle & =\left\langle\Delta \psi_{\dot{4}}\right\rangle=\frac{e^{-\phi / 2}}{2 \sqrt{3} b}\left(\Gamma^{12} \partial_{\dot{3}} \tau_{1}-\Gamma^{13} \partial_{\dot{2}} \tau_{1}\right)\left(1-\Gamma^{14}\right) \epsilon=0 \\
\left\langle\Delta \psi_{\dot{5}}\right\rangle & =\frac{\sqrt{b} e^{-\phi / 2}}{\sqrt{3}}\left(\gamma^{3} \partial_{\dot{3}} \tau_{1}+\gamma^{2} \partial_{\dot{2}} \tau_{1}\right)\left(1-\Gamma^{14}\right) \epsilon=0 \\
\left\langle\Delta \psi_{z}\right\rangle & =\frac{1}{2}\left(2 \partial-\frac{1}{2} \partial \ln \frac{e^{\phi}}{\tau}\left(i \Gamma^{23}\right)+\frac{1}{2} \bar{\partial} \ln \tau_{2} \Gamma^{523}-\partial \tau_{2}\left(i \gamma^{5}\right)\right) \epsilon=0 \\
\left\langle\Delta \psi_{\bar{z}}\right\rangle & =\frac{1}{2}\left(2 \bar{\partial}+\frac{1}{2} \bar{\partial} \ln \frac{e^{\phi}}{\tau}\left(i \Gamma^{23}\right)+\frac{1}{2} \bar{\partial} \ln \tau_{2} \Gamma^{523}+\bar{\partial} \tau_{2}\left(i \gamma^{5}\right)\right) \epsilon=0
\end{aligned}
$$

where $\left\langle\Delta \psi_{z}\right\rangle \equiv \frac{1}{2}\left\langle\Delta \psi_{\dot{2}}-i \Delta \psi_{\dot{3}}\right\rangle$ and $\left\langle\Delta \psi_{\bar{z}}\right\rangle \equiv \frac{1}{2}\left\langle\Delta \psi_{\dot{2}}+i \Delta \psi_{\dot{3}}\right\rangle$.

We find, by inspection, solutions to (23), (24), that is,

$$
\Gamma^{14} \epsilon=\epsilon .
$$

Furthermore, by the use of solution (19) and the form of (27), it is proved that eqs. (25), (26) lead to the following form:

$$
\begin{aligned}
& \left\langle\Delta \psi_{z}\right\rangle=\tau_{2}^{-1 / 4}\left(\frac{f}{\bar{f}}\right)^{ \pm 1 / 4} \partial\left[\tau_{2}^{1 / 4}\left(\frac{\bar{f}}{f}\right)^{ \pm 1 / 4} \epsilon^{ \pm}\right]=0, \\
& \left\langle\Delta \psi_{\bar{z}}\right\rangle=\tau_{2}^{-1 / 4}\left(\frac{f}{\bar{f}}\right)^{ \pm 1 / 4} \bar{\partial}\left[\tau_{2}^{1 / 4}\left(\frac{\bar{f}}{f}\right)^{ \pm 1 / 4} \epsilon^{ \pm}\right]=0,
\end{aligned}
$$

where $f \equiv f(z), \bar{f} \equiv f(\bar{z})$ and $\epsilon^{ \pm}$satisfy $i \Gamma^{23} \epsilon^{ \pm}= \pm \epsilon^{ \pm}$. Therefore the simultaneous solution to eqs. (23)-(26) is given by a linear combination of $\hat{\epsilon}^{+}$and $\hat{\epsilon}^{-}$,

$$
\hat{\epsilon}^{ \pm}=\tau_{2}^{-1 / 4}\left(\frac{f}{\bar{f}}\right)^{ \pm 1 / 4} \epsilon_{0}^{ \pm},
$$

where $\epsilon_{0}^{ \pm}$are constant spinors satisfying $\left(1-\Gamma^{14}\right) \epsilon_{0}^{ \pm}=0$ and $\left(-1 \pm i \Gamma^{23}\right) \epsilon_{0}^{ \pm}=0$.

Now we are led to the result that the background given by the cosmic string in our model has partially broken supersymmetry (i.e., supersymmetry associated with the restricted form of $\epsilon$ (30) remains unbroken). This conclusion is independent of the explicit functional form of the solution, since we have used only the holomorphicity of $\tau$ and the Einstein equations.

Next, we see this symmetry breaking from the point of view of the supersymmetry algebra. Generally speaking, the extended supersymmetry algebra has a central charge which is to give rise to partial symmetry breaking if the background has non-trivial charge (see refs. [8, 9] and references therein). If we concentrate on string-like solutions, we should define a supercharge per unit length and study the relation between the central charge (per unit length) and supersymmetry breaking. 
Since the vacuum configuration is translationally invariant in the $x^{4}$ direction in the present model, we consider the supercharge per unit length

$$
Q\left(\epsilon^{\prime}\right)=\int_{\partial \Sigma} \bar{\epsilon}^{\prime} \Gamma^{M N P} \psi_{P} d \Sigma_{M N}
$$

where $\Sigma$ is a three-dimensional space-like surface, which is given by the space spanned by coordinates $\left(x^{2}, x^{3}, \theta\right)$ in our model. The supersymmetry transformation, whose parameter is another spinor $\epsilon$, on $Q\left(\epsilon^{\prime}\right)$ is

$$
\delta_{\epsilon} Q\left(\epsilon^{\prime}\right)=\int_{\partial \Sigma} N^{M N} d \Sigma_{M N}=2 \int_{\Sigma} \nabla_{M} N^{M N} d \Sigma_{N}
$$

where

$$
N^{M N}=\bar{\epsilon}^{\prime} \Gamma^{M N P} \hat{D}_{P} \epsilon
$$

is Nester's form; here

$$
\hat{D}_{P}=\partial_{P}+\frac{1}{4} \hat{\omega}_{P A C} \Gamma^{A C}+\frac{1}{4 \sqrt{3}}\left(\Gamma_{P}^{Q R}+4 \gamma^{Q} \delta_{P}^{R}\right) \hat{F}_{Q R}
$$

is the supercovariant derivative.

In our case, the boundary $\partial \Sigma$ is considered as $S^{1} \times S^{1}$, where the former means the spatial infinity of the $x^{2}-x^{3}$ plane and the latter is the compactified dimension. And then we can rewrite (32) in terms of contour integrals. Assuming that the spinors $\epsilon^{\prime}, \epsilon$ are constant at spatial infinity (say, $\epsilon^{\prime} \rightarrow \epsilon_{0}{ }^{\prime}$, and $\epsilon \rightarrow \epsilon_{0}$ at spatial infinity) and the radius of the compact space $b$ is independent of the azimuthal angle $\varphi$ at spatial infinity $r \rightarrow \infty$ in the $x^{2}-x^{3}$ plane, we find

$$
\int_{\partial \Sigma} N^{M N} d \Sigma_{M N}=\epsilon_{0}^{\prime+}\left(M-Z \Gamma^{14}\right) \epsilon_{0},
$$

where $M$ and $Z$ can be interpreted as the mass per unit length of the string and the central charge, respectively. Explicit calculation reveals that $M$ and $Z$ take an identical value

$$
M=Z=\sqrt{\tau_{2}} \frac{3}{2} \pi n,
$$

where $n$ is the winding number of the string, defined as

$$
n \equiv \int_{\text {spatial infinity }} \frac{\partial \tau_{1}}{\partial \varphi} d \varphi .
$$

According to the expression (36), both $M$ and $Z$ diverge. To describe the result in terms of finite quantities, we must properly treat the length scale in the $x^{4}$ direction, that is, the factor $b^{-1 / 2}$ which appeared in (7). In other words, we find a finite result referring to the effectively four-dimensional "barred" metric system.

We observe here that $Z$ is proportional to

$$
\int \tilde{F}_{i j} d \Sigma^{i j}
$$


(where $i, j$ run over $1,2,3,5$ and means dual tensor), which is known to arise as a part of the central charge for generic $\mathcal{N} \geq 2$ supergravity [12].

By following the "standard" procedure $[8,9]$, we can find

$$
\delta_{\epsilon} Q(\epsilon)=\epsilon_{0}^{+}\left(M-Z \Gamma^{14}\right) \epsilon_{0} \geq 0 .
$$

Since our solution has $M=Z$,

$$
\begin{array}{ll}
\delta_{\epsilon} Q(\epsilon)>0, & \text { if } \Gamma^{14} \epsilon_{0}=-\epsilon_{0}, \\
\delta_{\epsilon} Q(\epsilon)=0, & \text { if } \Gamma^{14} \epsilon_{0}=+\epsilon_{0} .
\end{array}
$$

This fact coincides with the previous analysis on the transformation of fermionic fields; the above proves that if the central charge $Z$ is non-zero our background admits half-broken supersymmetry.

In future works we will clarify the symmetry among the Kaluza-Klein excited modes, using a similar technique as ref. [5].

The authors would like to thank A. Sugamoto for reading this manuscript. K.S. is indebted to Soryuusi shogakukai for financial support. He also would like to acknowledge financial aid of Iwanami Fūjukai.

\section{Note added}

Recently we have been informed of a string solution which involves non-zero torsion [13]. We thank Professor M. Hayashi for this information.

\section{References}

[1] A. Davidson and D. A. Owen, Phys. Lett. B155 (1985) 247; M. Yoshimura, Phys. Rev. D34 (1986) 1021; T. Koikawa and K. Shiraishi, Prog. Theor. Phys. 80 (1988) 108, and references therein.

[2] D. J. Gross and M. J. Perry, Nucl. Phys. B226 (1983) 29; R. D. Sorkin, Phys. Rev. Lett. 51 (1983) 87; B.-H. Lee, S. H. Lee, E. J. Weinberg and K. Lee, Phys. Rev. Lett. 60 (1988) 2231; M. Tomiya, J. Phys. G14 (1988) L153.

[3] A. Nakamula, S. Hirenzaki and K. Shiraishi, Nucl. Phys. B339 (1990) 533; A. Nakamula and K. Shiraishi, Mod. Phys. Lett. A5 (1990) 1109; Prog. Theor. Phys. 84 (1990) 1100.

[4] E. Cremmer, in: Superspace and supergravity, eds. S. Hawking and M. Roček (Cambridge U.P., Cambridge, 1980) p. 267.

[5] L. Dolan, Phys. Rev. D30 (1984) 2474. 
[6] E. Cremmer, B. Julia and J. Scherk, Phys. Lett. B76 (1978) 409.

[7] B. R. Greene, A. Shapere, C. Vafa and S.-T. Yau, Nucl. Phys. B337 (1990) 1; G. W. Gibbons, M. E. Ortiz and F. Ruiz Ruiz, Phys. Lett. B240 (1990) 50; S. Cecotti, Phys. Lett. B244 (1990) 23.

[8] A. Dabholkar, G. Gibbons, J. A. Harvey and F. Ruiz Ruiz, Nucl. Phys. B340 (1990) 33.

[9] A. Strominger, Nucl. Phys. B343 (1990) 167.

[10] Y. Hosotani, Phys. Lett. B126 (1983) 309; K. Shiraishi, Prog. Theor. Phys. 80 (1988) 601.

[11] A. Comtet and G. W. Gibbons, Nucl. Phys. B299 (1988) 719.

[12] G. W. Gibbons, in Proc. Heisenberg Symp., eds. P. Breitenlohner and H.P. Durr, Lecture Notes in Physics, No. 160 (Springer, Berlin, 1981).

[13] T. T. Fujishiro, M. J. Hayashi and S. Takeshita, Mod. Phys Lett. A6 (1991) 2237. 